\title{
Corela
}

Cognition, représentation, langage

HS-12 | 2012

Langue, espace, cognition

\section{Les prépositions ensemblistes et la question de leur emploi spatial}

\section{Emilia Hilgert}

\section{(2) OpenEdition}

\section{Journals}

Édition électronique

URL : http://journals.openedition.org/corela/2808

DOI : $10.4000 /$ corela.2808

ISSN : $1638-573 \mathrm{X}$

\section{Éditeur}

Cercle linguistique du Centre et de I'Ouest - CerLICO

Référence électronique

Emilia Hilgert, « Les prépositions ensemblistes et la question de leur emploi spatial », Corela [En ligne] HS-12 | 2012, mis en ligne le 19 décembre 2012, consulté le 20 avril 2019. URL : http:// journals.openedition.org/corela/2808; DOI : 10.4000/corela.2808

Ce document a été généré automatiquement le 20 avril 2019

\section{(c) (i) (2)(2)}

Corela - cognition, représentation, langage est mis à disposition selon les termes de la licence Creative Commons Attribution - Pas d'Utilisation Commerciale - Partage dans les Mêmes Conditions 4.0 International. 


\title{
Les prépositions ensemblistes et la question de leur emploi spatial
}

\author{
Emilia Hilgert
}

\section{Introduction}

1 Malgré un certain intérêt porté ces dernières années aux prépositions ensemblistes entre et parmi, ainsi qu'à la forme composée d'entre ${ }^{1}$ (les trois seront désignées désormais par Prép.ens), la description des multiples aspects de la relation spatiale ensembliste est loin d'être épuisée. Lorsque nous avons abordé la description de parmi dans une recherche plus ample sur la partition ( $c f$. Hilgert, 2007), nous avons proposé des critères permettant de distinguer les emplois ensemblistes des emplois spatiaux de cette préposition. Nous les avons comparés à ceux de entre, pour jeter une lumière nouvelle sur leur coexistence et sur la répartition de leurs emplois (cf. bibliographie). Dans cet article, nous souhaitons revenir sur un point qui semble être à l'origine du doute qui a plané assez longtemps sur la capacité de parmi à exprimer une relation spatiale. En effet, dans l'explication de cette relation spatiale spécifique, ce qui pose le plus de problèmes est la manière d'envisager le 'site' spatial ensembliste (est-il unique ou multiple?) et la capacité localisatrice d'un ensemble : s'agit-il d'une véritable localisation? Pour répondre à ces questions, nous nous appuierons sur quelques remarques antérieures sur l'emploi spatial des Prép.ens et nous apporterons une description du 'site' locatif de parmi (les N), en le mettant en contraste avec celui de entre (les $\mathrm{N}$ ) et en confirmant sa spatialité par des équivalences adverbiales. Celles-ci montreront les représentations multiples de l'espace avec parmi, produites par les différents paramètres de la relation spatiale 'A parmi B', à savoir la préposition fonctionnant comme un prédicat à deux arguments ${ }^{2}$, les configurations des entités localisée(s) et localisatrice(s) dénotées respectivement par SNA et SNB et le type de procès qui les relie. Chemin faisant, nous proposerons des solutions aux différents problèmes que posent les Prép.ens en espérant dissiper une certaine réserve à reconnaître leur emploi spatial. 


\section{Le microsystème des prépositions ensemblistes}

Les Prép.ens se combinent avec des SN pluriels ou comportant un nom collectif supposant des ensembles: parmi les voyageurs, parmi la foule. Ainsi, lorsque entre et d'entre sont ensemblistes ${ }^{3}$ comme dans vivre entre les Bulgares et revenir d'entre les morts, le repère spatial n'est pas duel (A entre B et C) : il n'est pas strictement vérifié par le cardinal deux, comme dans le passage entre deux maisons, le pays d'entre les [deux] fleuves ou la table est entre la porte et la fenêtre. Autrement dit, entre et d'entre sont ensemblistes lorsqu'ils se combinent avec des SNpl qui renvoient, explicitement ou par inférence, à plus de deux entités discrètes, ce qui les rapproche des emplois ensemblistes spécifiques à parmi. L'emploi ensembliste de entre n'annule pas, toutefois, son sens de base, à savoir une opposition duelle démultipliée, comme dans entre les mailles du filet ou filer entre les doigts.

Nous avons fait l'hypothèse, qui reste à confirmer par des études en diachronie, qu'au cœur du microsystème des Prép.ens se trouve entre, qui s'oppose à parmi et à d'entre, pour des raisons étymologiques et historiques (cf. Hilgert, 2010a). Alors que entre provient du latin inter ${ }^{4}$, parmi est une création du français ${ }^{5}$ qui a occupé progressivement certains emplois ensemblistes de entre. Cela expliquerait des emplois où elles génèrent des interprétations très voisines, correspondant à une zone grise où les différences entre elles ne sont plus très nettes ${ }^{6}$. Mais cela expliquerait surtout la répartition des emplois ensemblistes conformément à des sens très spécifiques à chacune de ces prépositions. Les différences de fonctionnement de entre et parmi peuvent être mises en relation avec des propriétés sémantiques qui les différencient dans leur association avec des pronoms. Dans ce cas, parmi ouvre l'ensemble vers une interprétation spatiale, alors que entre exprime la clôture catégorielle de l'ensemble, comme le montre l'opposition entre les énoncés suivants :

1. Pendant les pauses, les instits restent parmi eux [parmi les élèves]

2. Pendant les pauses, les instits restent entre eux [entre soi].

Le sens particulier de chacune de ces prépositions (parmi = dissociation interne de l'ensemble-site et localisation(s) aléatoire(s) d'entités-cibles d'une autre nature ; entre = fermeture relationnelle de l'ensemble avec une focalisation sur ses frontières catégorielles) intervient aussi dans leurs emplois spatiaux.

5 Pour distinguer les opérations sur des ensembles de la localisation ensembliste, rappelons que les premières s'appuient sur l'appartenance des entités $A$ et $B$ visées par la relation 'A Prép.ens B' à une même catégorie, impliquant parfois l'hyperonymie ou la possibilité d'indiquer l'identité de tel ou tel membre d'une classe ${ }^{7}$, alors que l'emploi spatial des Prép.ens est soumis à la contrainte de la différence catégorielle entre la cible et le site localisateur ${ }^{8}$. Ainsi, Parmi les drapeaux devant le Conseil de l'Europe on peut voir le drapeau de la France est un cas d'appartenance de A à l'ensemble B, signifiant que le drapeau de la France fait partie de l'ensemble les drapeaux qui se trouvent devant le Conseil de l'Europe, alors que Parmi les arbres on voit des maisons est un cas de localisation de A par rapport à l'ensemble B qui sous-tend la différence catégorielle arbres / maisons, tout comme dans Il y a des mauvaises herbes parmi les tulipes, qui s'interprète comme une localisation parce que les mauvaises herbes ne font pas partie de la classe des tulipes, les deux $\mathrm{N}$ étant des cohyponymes subordonnés au même hypéronyme plante. 


\section{L'ensemble localisateur comme site unique et types de localisations}

6 La première difficulté relevée dans la discussion de la spatialité de parmi les $N$ est celle de la pluralité des éléments qui servent de repères à l'entité localisée. C'est ce qui amène Borillo (1998: 15) à distinguer les cas d'unicité et de non-unicité du site. Comme le remarque l'auteure, l'unicité du site est sous-tendue par la plupart des prépositions et des verbes, parce qu'ils ont la propriété de mettre en jeu un seul repère spatial, comme dans La photo est sur la table. La non-unicité du site serait une sorte d'exception à la règle. Il s'agit, premièrement, de la relation ternaire entre une cible et deux sites exprimée par entre dans Le radiateur est entre la porte et la fenêtre (donc 'A Prép. B et C') et, deuxièmement, d'une relation :

«faisant intervenir plus de deux sites [qui] s'exprime à travers des prépositions comme entre, parmi, au milieu de, avec un nom pluriel (La photo se trouve parmi les papiers. La balle est nichée au milieu des orties) (Borillo, 1998 : 15).»

Le problème que pose ce dernier cas de figure est la multitude des repères spatiaux :

«Mais il est clair qu'au-delà de deux référents, la relation spatiale perd beaucoup

de sa précision et n'apporte que peu d'information sur les propriétés dimensionnelles ou orientationnelles des référents. »

8 Ce qui nous intéresse d'abord dans ce point de vue c'est que, si parmi, entre et au milieu de figurent dans les inventaires de Borillo, c'est parce que leur emploi spatial, si problématique qu'il soit, ne peut être ignoré. Leur marginalisation proviendrait de leur imprécision (la localisation est plus précise dans L'album photo est sur la table que dans L'album photo se trouve parmi les livres), et surtout du fait que les critères des relations spatiales impliquant des sites uniques leur seraient inappropriés. En effet, on ne peut plus appliquer à ce type de 'site' les propriétés spatiales des 'sites' uniques soumis à la variabilité de la perception / représentation que l'on se fait d'une entité du point de vue de la localisation (ou granularité variable): une, deux ou trois dimensions ou la représentation comme point, ligne, surface, volume; les plans vertical et horizontal; l'orientation intrinsèque ou contextuelle des objets sur l'axe vertical, horizontal, latéral ou frontal, etc., le tout se conjuguant dans une relation spatiale première, celle d'asymétrie entre la cible et le site.

9 Mais il y a plus : la distinction 'unicité vs non-unicité du site' permet à Borillo (1998) d'opérer une classification des relations spatiales statiques en deux sous-types. L'auteure distingue ainsi (i) les relations topologiques ou de localisation interne, lorsque la cible occupe une portion d'espace ou une 'place' qui a une certaine coïncidence avec le site, comme dans Le livre est sur la table, et (ii) les relations projectives ou directionnelles, c'està-dire de localisation externe, lorsque la cible se trouve dans une portion d'espace extérieure au site, comme dans aller vers la gare. Cette distinction se retrouve dans le classement des prépositions : entre et parmi sont donc considérées comme réalisant une localisation externe. Autrement dit, dans le cas de La photo se trouve parmi les papiers (qui ferait intervenir " plus de deux sites »), l'auteure analyse la relation entre la 'place' de la photo et la 'place' de chacun des papiers en question comme un cas de noncoïncidence spatiale : chaque individu 'papier' est considéré comme un site à part.

Or, comme nous l'avons déjà souligné (Hilgert, 2010b), le sens de cette phrase ne fait pas penser à la localisation de la photo à l'extérieur de chaque papier pris individuellement 
mais impose de considérer l'ensemble des papiers comme un site unique de référence, la cible étant localisée à l'intérieur de ce site ou dans ses limites9 ${ }^{9}$. L'emploi spatial des Prép.ens est, en effet, vérifié par la substitution du SP avec $y$ et en et par l'acceptation des questions avec où et d'où :

(3) a) On a trouvé cet album photo parmi les livres de Jean. // Où ça ? Parmi les livres de Jean.

b) On a fouillé parmi les livres de Jean et on $y$ a trouvé cet album photo. ( $y$ = parmi les livres de Jean)

(4) a) On a fait poser des bancs entre les arbres qui bordent l'allée. // Où ça ? Entre les arbres.

b) Comme il y avait de la place entre les arbres qui bordent l'allée, on y a fait poser des bancs. $(y=$ entre les arbres).

(5) a) Les soldats étaient prêts à lancer des grenades sur les premiers ennemis qui sortiraient d'entre les arbres. // D'où sortiraient-ils ? D'entre les arbres.

b) On s'est dit qu'il pourrait y avoir des ennemis qui pourraient sortir d'entre les arbres et les soldats étaient prêts à lancer des grenades sur les premiers qui en sortiraient. (en = d'entre les arbres)

11 L'application des tests syntaxiques classiques prouve qu'il y a bien un emploi spatial de ces prépositions, les différences interprétatives venant des particularités sémantiques de chacune. Mais quelle représentation se fait-on globalement des sites désignés par Prép.ens + SN ? Si l'on pense aux représentations langackeriennes utilisées par Gréa (2005) pour la lecture collective d'au milieu de / parmi les étoiles, il est vrai que l'on vise l'espace entre les étoiles (extérieur à chaque étoile pourvue de limites intrinsèques ${ }^{10}$ ), mais il est tout aussi vrai que cette localisation se fait dans les limites floues, non ontologiques, non intrinsèques, mais spatiales de l'ensemble qu'elles forment: $\mathrm{si}$, dans le cas d'une photo qu'on a trouvée parmi les papiers, la photo n'était pas au milieu des papiers, elle serait localisée à côté des papiers ou à l'extérieur du tas de papiers. L'inférence des limites intrinsèques des éléments d'un ensemble (des entités concrètes discrètes) n'exclut pas la perception et la représentation d'un ensemble comme occupant un espace dont on pourrait tracer les limites (cf. la ligne sinueuse qu'utilise Gréa, 2005, à la suite de Langacker, 1987, pour délimiter un regroupement d'étoiles).

On peut, effectivement, surmonter le premier problème de l'interprétation spatiale des Prép.ens, celui du type de site, unique ou multiple, et donc le type de localisation qu'elles expriment, interne ou externe, si l'on prend en considération ces deux types de limites: les limites intrinsèques des entités discrètes et les bornes de l'ensemble qui a nécessairement une dimension spatiale. Il est donc plus naturel de considérer un ensemble quelconque les $N$ comme un site unique formé d'éléments dissociés préservant entre eux des intervalles de différentes dimensions et dispositions permettant la localisation d'une ou de plusieurs entités dans cet 'espace'. C'est une interprétation qui s'accorde avec la définition du N espace donnée par Huyghe (2009: 173) : «Dans le monde physique, un espace dénote une portion du vide qui s'intercale entre les objets » et par Kleiber (2009: 19), qui explique que la perception visuelle des entités dénotées par espace est essentiellement impliquée par la perception visuelle des entités matérielles qui le délimitent :

"Ce sont les frontières, donc les formes des objets matériels qui supposent de l'audelà externe, ou du vide, appelé espace. Si l'on voit qu'il y a un intervalle entre $\mathrm{x}$ et $z$, c'est parce que les limites de l'intervalle spatial en question appartiennent aux objets $\mathrm{x}$ et $\mathrm{z}$, et c'est leur perception visuelle qui permet de "voir" l'espace qu'il y a entre elles. » 
13 La relation spatiale 'A parmi B' comme dans il y a des arbres parmi les maisons suppose qu'entre les entités maisons il y a des portions d'espace que celles-ci découpent grâce à leurs limites intrinsèques, mais suppose aussi que la pluralité les maisons (ou la " collection », dans les termes d'Aurnague, 2004 : 56) a une frontière donnée par l'étendue de cet ensemble dans l'espace : on sait pertinemment quand on est sorti d'une forêt ou quand on a quitté un quartier. Les limites intrinsèques des entités individuelles ne contredisent pas le bornage spatial d'un groupement qu'elles peuvent former. La limite venant du fait qu'il s'agit d'un ensemble d'entités du même type (désignées par le même $\mathrm{N}$ ), on se représente ce groupement comme un site spatial unique, s'il sert de localisateur à des entités d'une autre catégorie.

Il faut reconnaître que la prise en compte des bornes spatiales données à l'ensemble localisateur par sa propriété de collection d'entités du même type a quelque chose de paradoxal dans l'expression de la localisation avec Prép.ens. Même si l'on sait qu'un ensemble (ou une " collection ») de maisons occupe de l'espace, ce n'est pas la frontière de ce groupement qui intéresse la localisation avec les Prép.ens : ce qui est perçu ou représenté par la relation 'A Prép.ens B' (des arbres parmi les maisons) c'est l'espace que délimitent entre eux les éléments dissociés de l'ensemble (les maisons), ce qui rend les frontières de la collection floues ( $c f$. Kwon-Pak, 2006). On a toutefois besoin de la notion de 'limite de l'ensemble' et de la notion de 'site unique ensembliste' pour décrire les différentes localisations réalisées par les Prép.ens, pour pouvoir distinguer entre elles les différentes localisations internes exprimées par parmi et entre et décrire celle réellement externe ou projective opérée par d'entre. Autrement dit, comme la localisation de A par rapport à B dans les relations A parmi B / A entre B se fait dans les intervalles qui existent entre les éléments de l'ensemble $B$, elle est interne ou interne - médiane parce qu'elle se fait dans les limites de cet ensemble. En revanche, la forme composée d'entre représente le seul cas de localisation externe par rapport à un ensemble ${ }^{11}$ : renforcée par la présence d'un verbe de déplacement, l'entité Asg./pl. est perçue comme franchissant les limites spatiales de l'ensemble B, comme quittant l'espace délimité par cet ensemble.

Nous distinguerons donc désormais le site 'single', constitué d'une entité discrète (par exemple dans une armoire), et le site ensembliste unique, comme dans parmi / entre / d'entre les papiers. Pour ce dernier type, les interprétations se comprennent toutes comme des localisations de type 'environnement' ou 'milieu', mais manifestent des degrés qui vont de la localisation précise à une localisation plus vague, en fonction de la perception ou de la représentation que l'on se fait de l'ensemble localisateur (compact, ordonné ou éparpillé) et des intervalles qui existent entre les éléments dissociés de l'ensemble (plus réduits ou plus grands) ou de la disposition de ces éléments (en tas, en rangée, en groupement plutôt dispersé, etc.).

16 Précisons avant de clore l'argumentation en faveur du site ensembliste unique que, dans le cadre de la localisation interne ou environnementale exprimée par entre les $N$ et parmi les $N$, chaque préposition crée des interprétations qui lui sont particulières $(c f$. pour leur mise en contraste, Hilgert, 2010a et b) :

(i) la localisation du type 'environnement' avec parmi est perçue comme indéterminée, aléatoire, induisant une occupation partielle des intervalles ;

(ii) a contrario, la localisation du type 'environnement' avec entre est perçue comme étant plus précise parce que entre signifie l'ordonnancement d'un ensemble, l'individualisation de ses éléments, transformés en balises qui permettent de structurer l'espace sous forme d'interstices, de parcours pointant à chaque balise, d'intervalle entre deux ensembles munis chacun de frontières. 
17 D'entre peut s'opposer aux deux. Il s'oppose à entre ordonnateur de dualités démultipliées, comme dans Le plâtre est tombé d'entre les pierres (Flaubert) vs Il y avait du plâtre entre les pierres. Malgré son lien morphologique avec entre, d'entre peut s'opposer du point de vue sémantique à parmi dissociateur ensembliste, comme dans la succession de titres extraits de Candide de Voltaire: Ce que devint Candide parmi les Bulgares (chapitre second) et Comment Candide se sauva d'entre les Bulgares (chapitre troisième) et non *de parmi les Bulgares. Cette opposition sémantique invite à croire que parmi a pris la place de entre dans l'expression de la localisation ensembliste indéterminée du type 'environnement' ou 'milieu'12, correspondant au français classique Les médecins vivent bien entre les malades (Montaigne, I, 274, in Littré).

\section{La représentation de l'espace réalisée par le SP parmi les $N$}

Les différentes valeurs spatiales de parmi ressortent de l'interaction forte entre la préposition et son co-texte dans la relation 'SNA Prép. SNB' où SNB réfère à un ensemble. La configuration de la cible et du site, en rapport avec différents types de procès, crée des valeurs de localisation, déplacement et environnement, qui seront observées sur des exemples de Frantext et mises en évidence par des équivalences adverbiales.

\subsection{Localisation statique et dynamique parmi les $N$}

19 La localisation de la cible singulière (SNA-sg) par rapport à l'ensemble est statique individualisée et indéterminée, équivalente à quelque part. Dans ce cas, le SP en parmi exprime un espace non saturé, qui correspond au sens 'partitif' de l'adverbe quelque part, expliqué par Gerhard-Krait \& Kleiber (2006: 90). Il signifie une occupation partielle d'un site, les énoncés avec quelque part donnant lieu à des inférences du type "pas partout ", dans un seul endroit indéterminé. Huyghe (2009: 135) identifie aussi un sens 'partitif' dans le nom endroit qui «s'emploie régulièrement dans une perspective locative globale, lorsqu'on vise un site au sein d'un ensemble particulier, borné ou non ", comme c'est le cas dans l'exemple suivant :

(6) Un peu plus haut, au pied des remparts du couvent Na Slovanech, sur une petite butte plantée de lilas blancs et mauves, la géante apparut. Elle se tenait parmi les arbres, dissimulée par les branchages. On ne pouvait pas distinguer son visage, masqué par une grappe de lilas blanc. On n'apercevait que des pans de son corps. Elle se confondait presque avec l'ombre violâtre du bosquet. Elle se tenait très droite, et immobile, une main appuyée contre un tronc ... (Germain S., La Pleurante des rues de Prague, 1992, p. 48)

20 Avec des cibles plurielles (SNA-pl) statiques, la localisation est comprise comme un éparpillement, correspondant au sens de çà et là, ici et là, à plusieurs endroits indéterminés, signifiant plusieurs places occupées indéterminées dans une occupation partielle du site :

(7) Traversée des régions meurtries par la guerre. Quelques ruines encore parmi les maisons neuves. (Gide A., Journal, 1939, p. 935)

(8) On tournait le dos à la mer. En face, c'étaient les collines râpeuses qui tendaient leurs dos. Il y avait quelques maisons, éparpillées parmi les arbres, avec des champs de vignes, des oliviers, des terrasses. (Le Clézio J.-M.-G., Le Déluge, 1966, p. 238) 
troisième cas de figure est celui de la cible individuelle animée (SNA-sg-ego) qui est présentée en déplacement indéterminé à l'intérieur de l'ensemble, correspondant au sens de se déplacer par là :

(9) Voiture arrêtée; sous-bois où je suis allé attendre l'accalmie... J'ai dû pénétrer assez loin parmi les arbres avant de m'abriter sous un chêne, car la pluie tombait de plus en plus fort... (Sollers P., Le parc, 1961, p. 136)

Une pluralité animée (SNA-pl) peut être présentée en déplacement individuel indéterminé faisant penser à des trajets multiples. Cette valeur est équivalente à par-ci par-là, qui s'accorde avec le verbe se disperser :

(10) Il s'en assura en emmenant la meute dans ses tournées de recrutement. A l'entrée d'un village les chiens se dispersaient parmi les maisons et les jardinets ...

(Tournier M., Le Roi des Aulnes, 1970, p. 458)

Parmi exprime l'idée de mélange hétérogène de deux ou plusieurs ensembles parce qu'on ne peut se représenter, dans l'exemple précédent, une rangée de maison en face d'une rangée de jardinets, idée qui correspond en revanche à entre. Avec deux SN, entre (et non parmi) transmet l'idée d'un intervalle de localisation d'une entité entre les deux ensembles, ce qui est montré par l'acceptation de marqueurs adverbiaux, alors que parmi confond les deux ensembles, rejetant les adverbes de localisation séparée :

(11) Le journaliste se trouvait entre les députés et les syndicalistes. / Le journaliste se trouvait entre les députés, à sa gauche, et les syndicalistes, à sa droite.

(12) Le journaliste se trouvait parmi les députés et les syndicalistes. / *Le journaliste se trouvait parmi les députés, à sa droite, et les syndicalistes, à sa gauche.

(13) Les chiens couraient entre les maisons et les jardinets. [dans l'intervalle entre les maisons, d'un côté, et les jardinets, de l'autre]

Enfin, dans la localisation exprimée à l'aide de parmi les $N$, les $N$ peut être interprété comme un attribut d'un site 'single'. C'est le cas lorsque la localisation regroupe un site 'single' et un site 'collection' désignés respectivement par un SPsg localisateur ayant des propriétés spatiales plus nettes (une autre préposition, un SN singulier) et par un SPpl en parmi, comme dans :

(14) a) Quelques jours plus tard, en mettant de l'ordre dans son secrétaire, je trouvai parmi le papier à lettres, les timbres, les enveloppes doublées de soie, les rouleaux de scotch, les cartes de visite et le courrier en attente, un document unique... (Flem L., Comment j'ai vidé la maison de mes parents, 2004, p. 81)

b) Quelques jours plus tard, en mettant de l'ordre dans son secrétaire, parmi le papier à lettres, les timbres, les enveloppes doublées de soie, j'y trouvai un document unique (y1 = dans son secrétaire ? y2 = parmi le papier à lettres, les timbres, etc. ? y3 = dans son secrétaire, parmi le papier à lettres, les timbres, les enveloppes)

Si l'on est tenté de faire une pronominalisation en $y$, on pourrait considérer le SP dans le secrétaire comme un site 'single' plus conforme à l'idée de localisation que le site ensembliste le papier, les timbres, les enveloppes et donc comme un attracteur de la substitution $\left(y_{1}=\right.$ dans son secrétaire). Or, une interprétation plus attentive aboutit à l'idée que le premier site englobe le deuxième, ce qui les rend difficilement dissociables. D'autre part, la localisation de la cible 'un document' ne peut être qu'unique, dans les limites de la place qu'elle occupe; $y_{3}$ renvoie alors simultanément aux deux emplacements $y_{1+} y_{2}$ dans une sorte de zoom de focalisation de l'un vers l'autre ${ }^{13}$, réalisant ce que Vandeloise (1988) appelle des «localisations emboîtées» exprimées par des SP juxtaposés à contenus sémantiques différents (cf., pour le même type d'interprétation de SP juxtaposés en par et à travers, Stosic, 2005).

Corela, HS-12 | 2012 
Avec entre les $N$, on peut avoir des configurations voisines d'un environnement et le contraste avec parmi les $N$ semble minime, parce que les énoncés qui suivent acceptent également les gloses par là pour le cas suivant :

(15) Le bois avance vers nous, nous y sommes en fin d'après-midi : faux et faucilles battent maintenant les ronces de l'orée du bois. Les outils déposés, nous courons entre les arbres pleins de rayons, nous crions de plus en plus fort pour que l'écho, venu d'en bas, du fond, dure plus longtemps. (Guyotat P., Formation, 2007, p. 192)

et çà et là ou par-ci par-là pour le cas énoncés suivants :

(16) Nous montons à Chaussitre, montagne pelée, où le granit affleure, entre les genévriers, à onze cents mètres : vers le haut, une coulée de basalte, où est empreint le contour d'un sabot de cheval. (Guyotat P., Formation, 2007, p. 177)

(17) Nous avons mâchouillé des artichauts crus et calmé l'agacement ferreux des dents en buvant du chasselas pressé ou du sirop d'orgeat. Nous avons cherché entre les rocailles le rare pèbre d'ail et j'ai pu démontrer comment la graine d'avoine se déguise en cuisses de sauterelle ... (Szczupak-Thomas Y., Un diamant brut, 2008, p. 327)

Pourtant, malgré leur interchangeabilité dans certains cas (nous courons entre / parmi les arbres; le granit affleure entre / parmi les genévriers), entre et parmi ne créent pas le même sens. La différence entre ces deux prépositions est prouvée par le fait qu'au moins le deuxième contexte accepte l'addition de l'adverbe partout signifiant une saturation des intervalles qui se forment entre les arbres pris deux par deux: le granit affleure partout entre les genévriers vs *le granit affleure partout parmi les genévriers. Avec entre, c'est l'idée d'intervalles multiples qui est signifiée par courir entre les arbres, une saturation possible de ces intervalles dans le granit affleure entre les genévriers et des interstices serrés dans chercher le pèbre d'ail entre les rocailles.

Il y a, enfin, des énoncés où figurent les deux prépositions entre et parmi, qui peuvent être interchangeables, mais où la différence interprétative, si minime qu'elle soit, est due au sémantisme de chacune d'entre elles :

(18) a) [vue en plongée sur un port new-yorkais et les jetées transatlantiques] Parmi les montagnes de balles, entre les caisses à inscriptions françaises, se faufilent les femmes nu-tête, souriantes, en manteau du soir, les fracs, les chapeaux... (Morand P., NewYork, 1930, p. 204)

b) Entre les montagnes de balles, parmi les caisses à inscriptions françaises, se faufilent les femmes nu-tête, souriantes, en manteau du soir, les fracs, les chapeaux.

On peut associer aux SP entre les $N$ une idée d'alignement, d'ordonnancement des $\mathrm{N}$, alors que les SP parmi les $N$ donnent plutôt une idée d'éparpillement, de désordre, d'un passage au hasard. Cette interprétation va au-delà de la simple variation stylistique. Comme l'explique Stosic (2005: 215), l'interchangeabilité, dans certains contextes, de prépositions a priori considérées comme synonymes ne vient pas de leur synonymie, mais résulte de «la capacité des humains de conceptualiser une même configuration spatiale sur la base de concepts différents. »

Dans les emplois analysés, signifiant une localisation statique et différentes localisations dynamiques, le sens spatial de parmi regroupe ceux de ses constituants, par et mi. Dans l'expression de la relation spatiale dynamique, l'élément par apporte l'idée de 'trajet', comme le montrent Aurnague \& Stosic (2002) et Stosic (2005) : par exprime une relation spatiale dynamique en introduisant le site médian que l'entité-cible parcourt dans son déplacement d'un site initial à un site final (comme dans passer par la fenêtre / par le bois / par les dunes / par le territoire chinois). Il intervient donc essentiellement dans l'expression du 'trajet' à travers des entités-sites " possédant des portions d'espace indispensables 
pour que le passage d'une zone à une autre puisse se réaliser » (Stosic, $2005: 215)$. Dans le cas d'un déplacement parmi les $N$, le site de parmi est un ensemble d'éléments préservant des espaces entre eux et un 'trajet' imaginé au milieu de ce site (sens de mi) se fait par une succession de séquences spatiales : on passe de $x$ à $z$ par $y$ et, une fois arrivé à $z$, on réalise un passage de $z$ à $q$ par $p$, ainsi de suite, dans les limites de l'ensemble localisateur. Cela ne veut pas dire que les espaces ainsi modélisés se succèdent naturellement dans cet ordre, comme le note Kwon-Pak (2006), mais qu'ils sont fonctionnellement ordonnés ainsi ( $x>y$ $>\mathrm{z}>\mathrm{p}>\mathrm{q}$ ) par le déplacement aléatoire de la cible, supposant qu'il y d'autres espaces dans la dimension spatiale de l'ensemble qui ne sont pas parcourus. Cela revient à dire que le trajet que sous-tend parmi correspond plus à une traversée comme celle désignée par à travers, qui exprime, comme l'explique Stosic (2005), l'idée d'aménagement d'un chemin en choisissant certaines de ses portions d'espace, correspondant à l'idée de se frayer un chemin. Avec un SN singulier, à travers signifie un passage frayé dans des sites non aménagés pour le déplacement. Avec un $\mathrm{SN}$ pluriel, à travers renvoie à un réseau de passages potentiels que la cible peut emprunter au cours de son déplacement. L'idée de 'passage frayé' aléatoire rapproche à travers de parmi.

Dans la localisation statique parmi les $N$, cette relation spatiale signifie que visuellement on perçoit la ou les entités-cibles dans une disposition aléatoire dans les espaces préservés entre eux par les éléments de l'ensemble-site, ou qu'on peut les percevoir de manière partielle, en partie cachées par les éléments de l'ensemble. Le 'passage frayé' correspond alors au cheminement de notre perception qui discerne les limites des éléments de l'ensemble-site permettant de voir ou d'entrevoir les entités-cibles.

\subsection{Parcours, itinéraire, chemin... parmi les $N$}

Parmi peut exprimer l'idée de 'parcours' d'une manière qui lui est spécifique : si entre signifie que le procès relie entre eux les éléments de l'ensemble, avec un pointage explicite ou inféré de chacun, en passant de l'un à l'autre (comme dans le coursier faisait la navette entre les services), parmi signifie un parcours dans les intervalles sans individualiser les éléments de l'ensemble, c'est-à-dire qu'il signifie moins " en passant de l'un à l'autre " qu'une traversée médiane au hasard. L'idée de 'parcours' dans un environnement est produite par le verbe marcher ou par les $\mathrm{N}$ sentier, chemin, route, itinéraire dont on comprend, grâce à parmi les $N$, qu'ils ne sont pas balisés (cela s'accorderait mieux avec entre les $N$ ) mais plutôt aléatoires. Nous avons choisi d'observer les représentations que l'on se fait des déplacements ou des itinéraires dans un site de même nature les arbres, pour montrer les gloses adverbiales appropriées à chaque cas de traversée signifiée par parmi, en prenant comme seul critère variable la cible unique ou multiple, animée [+humain] ou inanimée (le N chemin), statique ou en mouvement. Dans le premier cas de figure, une cible unique le sentier ou la route / chaque tournant (SNA-sg-inanimé) marque, par son sens, un parcours indéterminé dans un décor d'arbres, parmi les $N$ ayant comme équivalent par là-bas ou, plus précisément, dans cet environnement :

(19) Au-delà des rails, les bois commençaient. Mais, il trouva le sentier sans hésiter. Il montait parmi les arbres. Les lumières de la ville se montrèrent à nouveau, pardelà la vallée où s'enfuyait la voie du chemin de fer. (Coindreau M.-E., Lumière d'août , 1935, p. 152)

(20) Puis la route s'engagea par lacets dans un cirque de roches rouges que chaque tournant parmi les arbres faisait plus nombreuses et plus belles, plus claires aussi avec le jour. (Bonnefoy Y., Rue Traversière et autres récits en rêve, 1987, p. 190) 


$$
\begin{aligned}
& \text { déplacement. Les gloses possibles sont une fois encore par là-bas et dans cet environnement, } \\
& \text { comme dans : }
\end{aligned}
$$

(21) La bouche parfumée de menthol, il marche d'un pas allégé. Il tourne autour de chaque pilier de la tour Eiffel, s'invente des itinéraires parmi les arbres. Il se sent mieux, presque délivré. (Sabatier R., Le Chinois d'Afrique, 1966, p. 44)

(22) Ils avaient quitté le sentier et marchaient parmi les arbres clairsemés, longeant la palissade de pieux. (Oldenbourg Z., Les Cités charnelles ou l'histoire de Roger de Montbrun, 1961, p. 209)

Un SNA-sg signifie un parcours sinueux et évanescent dans les intervalles créés par les arbres, que l'on perçoit comme contigus. Cet emploi peut se gloser par (là-bas), au milieu $\operatorname{des} N$ :

(23) Le chemin suivait les creux des dunes, se perdait au loin parmi les arbres, de petits chênes tourmentés et rabougris, mutilés, tronqués, dépouillés, dans leur lutte perpétuelle contre le vent de mer... (Van Der Meersch M., L'Empreinte du dieu, 1936, p. 142)

Globalement, l'idée de parcours donnée par des $\mathrm{N}$ dénotant des chemins s'accompagne de celle d'évolution de ceux-ci dans un environnement, 'par là-bas'. Une comparaison avec entre les $N$ qui renvoie lui aussi à un passage dans un environnement montre que entre influence l'interprétation par sa précision spatiale :

(24) Beaucoup de tunnels, une locomotive qui, à des heures régulières, rejette beaucoup de fumée et siffle et dont on suit le parcours entre les sapins jusque loin audessus de nous. (Guyotat P., Formation, 2007, p. 88)

Cette fois, même si l'on comprend que le parcours entre les sapins est un parcours dans un environnement de sapins, le sens de entre nous fait nous représenter un ordonnancement plus précis. Il corrobore l'idée qu'une voie ferrée ne peut avoir le cheminement aléatoire, étroit et sinueux d'un sentier qui se fond dans le milieu : à l'inverse, elle délimite plus précisément un tracé aisément repérable. Avec parmi, la focalisation sur le tracé s'estompe, laissant réapparaître la notion d'« environnement " comme le souligne le verbe se perdre de l'exemple (23).

\subsection{Vivre dans un environnement du type parmi les $\mathbf{N}$}

Avec des verbes comme être Adj., être élevé, vivre, le ou les repéré(s) (SNA-sg/pl-ego) sont des humains qui évoluent dans différents environnements, la seule glose possible étant au milieu des $N$, dans cet environnement particulier. Il ne s'agit plus d'une localisation à proprement parler, dans le sens où l'environnement n'est plus représenté comme un attribut d'un possible site 'single' du type tiroir, parc, forêt, montagne, etc., mais d'une focalisation sur l'environnement dans lequel évoluent un ou des individus :

(25) Je pense aussi que l'étude de la nature ne nuit en aucune façon à la pratique de la vie, et que l'esprit qui sait être libre et ailé parmi les oiseaux, parfumé parmi les fleurs, mobile et vibrant parmi les flots et les arbres, haut, serein et paisible parmi les montagnes, sait aussi, quand vient l'heure, et mieux peut-être que personne, être intelligent et éloquent parmi les hommes. (Hugo V., Le Rhin: lettres à un ami, 1842, p. 311)

(26) Car tout en eux prenait des accents de colère, même l'amour. Ils avaient été élevés davantage parmi les arbres que parmi les hommes, ils s'étaient nourris depuis l'enfance des fruits, des végétaux et des baies sauvages qui poussent dans les sousbois ... (Germain S., Jours de colère, 1989, p. 84) 
(27) Seules, la couleur et l'odeur la séduisaient. C'était vrai d'ailleurs que ça lui allait bien de vivre parmi les fleurs. Elle-même avait un teint de lys. Comment faisait-elle, avec tout l'alcool qu'elle pintait? Pas une couperose. Rien. (Guérin R., L'Apprenti, 1946, p. 283)

\subsection{Vivre dans un milieu du type parmi les $N$}

38

Avec le même type de verbes (vivre, se retrouver, être né et élevé), des humains peuvent être représentés comme évoluant dans un milieu d'humains, si le N du SN introduit par parmi désigne différentes classes d'humains (hommes, hommes d'action, indigènes, Indiens, passants, etc.). Le SP en parmi renvoie alors à un 'milieu' :

(28) La culture était alors littéraire, car elle consistait moins à accroître la base, alors immuable, des connaissances positives qu'à connaître et comparer les opinions et les émotions des philosophes et des poètes. Elle était humaniste, parce qu'elle apprenait à vivre parmi les hommes et à les comprendre. (Cros L., L'Explosion scolaire, 1961, p. 113)

(29) Madeleine avait appris à juger vite, d'un coup d'œil, à force de vivre parmi les hommes d'action. L'entrée de Jean Renault la laissa, cependant, hésitante. (Déon M., La Carotte et le bâton, 1960, p. 59)

(30) [...] elle m'accueillait toujours - c'était chez elle une sorte d'automatisme - par un «Comment ça va? » qui me gênait affreusement. Comme on peut avoir honte parfois d'être bien-portant, d'avoir faim, de respirer sans même s'en apercevoir, et de devoir refouler l'impatience de se retrouver parmi les passants qui vont et viennent ! (Pontalis J.-B., L'enfant des limbes, 1998, p. 45)

(31) L'expérience d'une jeune Autrichienne qui a été vivre parmi les Indiens Wayapi en Guyane est révélatrice. Les premiers mois, ils toléraient sa présence mais semblaient se désintéresser d'elle. En fait, elle était en observation. Ils prenaient le temps de l'éprouver. (Dolto F., La Cause des enfants, 1985, p. 84)

(32) [...] ce témoignage à tous ceux, provenant d'autres régions du monde, qu'on aurait pu citer dans le même sens, parce qu'il émane d'un ethnologue né et élevé parmi les indigènes, parlant couramment leur langue, et qui leur est resté profondément attaché. (Lévi-Strauss C., La Pensée sauvage, 1962, p. 282)

L'enveloppement du type 'environnement' ou 'milieu' exprimé par parmi les $N$ n'est plus à proprement parler spatial, mais plutôt relationnel : il y a une action du milieu ou de l'environnement sur la 'cible', qui suppose une influence de celui-ci, négative ou positive, un transfert, un échange, voire un mimétisme.

\subsection{Bilan}

Les gloses adverbiales des localisations du type 'environnement' signifiées parmi les $N$ sont :

- $y$, là, avec un sens spatial assez proche de la spatialité des sites 'single'

- quelque part, statique individuel, en un lieu quelconque, signifiant une occupation partielle de l'espace

- ça et là, ici et là, statique pluriel, créant une idée de dispersion, d'éparpillement en désordre

- par-ci par-là, par là-bas, dynamique, individuel et pluriel, exprimant une idée de mouvement en divers endroits et donc une occupation transitoire, partielle et indéterminée de l'espace

- au milieu de, transmettant une idée d'environnement ou de milieu, ayant le sens 'dans cet environnement', parfois en tant qu'attribut d'un site 'single' standard

- dans cet environnement, dans ce milieu, avec des prédicats du type vivre, être élevé, se reposer, etc. 


\subsection{Analyse de 'A parmi B'}

41 'environnement' et permettent une analyse de la localisation environnementale de parmi en étapes. Dans la relation spatiale 'A parmi B', quatre paramètres entrent en jeu : le sens de la préposition, la configuration du deuxième argument (le sens du $N \mathrm{du} S \mathrm{SN} B$ ), la configuration du premier argument (le sens du $N$ du SNA) et, enfin, le sens du verbe de l'énoncé comprenant 'A parmi B'. Il en résulte une localisation aléatoire dans les intervalles, n'excédant pas les limites de l'ensemble.

Parmi signifie d'abord qu'il y a des intervalles ou des espaces entre les individus ou les éléments qui constituent l'ensemble les $N$ et que ce sont ces espaces internes à l'ensemble qui concernent la localisation d'une entité ou d'entités concrètes d'une autre catégorie. Mais toute la difficulté provient de la configuration de ces espaces ou de ces 'vides' spatiaux occupables et de la représentation que l'on s'en fait selon le sens du SN introduit par parmi, parce que les différents types de localisation dépendent de la configuration et des dimensions de ces espaces, qui, eux, auront la configuration, la forme, les dimensions que leur donnent les entités concrètes qui les délimitent. Parmi les $N$ signifie alors que :

i. dans l'ensemble dénoté par les $N$ il n'y a pas nécessairement de passage ordonné préconstitué qui traverse l'ensemble ou qui l'ordonne;

ii. les espaces délimités par les éléments de l'ensemble peuvent ne pas être réguliers ou équivalents entre eux, ou avoir les mêmes dimensions ;

iii. ces espaces se succèdent ou se fondent l'un dans l'autre, dans un continuum de forme indéterminée, offrant une multitude de possibilités de localisation ou de déplacement dans ces intervalles présentant un 'enchevêtrement' aussi abstrait que le $\mathrm{N}$ espace: visible, mais n'ayant pas de matérialité, celle-ci provenant des éléments qui le délimitent ;

iv. la configuration de ce continuum spatial est multiforme voire protéiforme, c'est-à-dire qu'elle peut être envisagée de maintes manières, en fonction du point de vue de l'énonciateur, en fonction des limites et de la disposition des entités concrètes qui le délimitent ;

v. l'énonciateur peut être situé à l'extérieur de l'ensemble de localisation ou avoir un point de vue englobant l'ensemble ou une grande partie de celui-ci : lorsqu'il dit qu'il y a des ruines parmi les maisons, il doit embrasser de la vue un tableau suffisamment large pour voir et les maisons et les ruines, avoir un point de vue extérieur à ce champ de vision ; inversement, dans le cas de vivre, être élevé parmi les $\mathrm{N}$, c'est-à-dire évoluer dans un environnement ou dans un milieu particuliers, le point de vue est interne à cet environnement, et donc à l'ensemble les $N$;

vi. la localisation par rapport à un ensemble d'entités discrètes dissociées est interne au site, se fait dans les limites de ce site, dans le continuum spatial multiforme et protéiforme délimité par des entités concrètes en nombre (Cadiot 2002 appelle cette localisation 'médiane', c'està-dire 'dans les limites / à l'intérieur de l'ensemble') ;

vii. la localisation dans un tel site est bien réelle mais elle est indéterminée; parmi signifie l'exploration de l'espace protéiforme, un trajet linéaire mais sinueux, qui peut se recouper, ou un éparpillement aléatoire dans ce continuum spatial protéiforme; la localisation peut varier selon le co-texte de parmi: la localisation est principalement indéterminée, correspondant, selon le cas, aux adverbes là, quelque part, quelque part par là, çà et là, ici et là, par là, par ci par là. 
La comparaison de parmi avec entre ensembliste, dans les emplois où entre semble avoir la signification la plus proche de celle de parmi, révèle des différences non pas syntaxiques mais interprétatives entre ces prépositions. Entre a un sens de 'séparateur - ordonnateur' des éléments de l'ensemble, avec une idée d'intériorité et de saturation possible de tous les intervalles. Parmi localise de manière indéterminée une entité ou un parcours dans son site ensembliste. Entre signifie soit des intervalles multiples formés par les éléments de l'ensemble pris deux à deux, saturés, c'est-à-dire tous occupés, soit un intervalle unique, formé par une disposition ordonnée des éléments de l'ensemble en deux rangées ou deux groupes; parmi garde dans ses emplois spatiaux son sens de l'expression linguistique des opérations sur des ensembles, à savoir la disposition 'partitive' des éléments de l'ensemble, autrement dit leur dissociation interne et leur disposition aléatoire. Les deux prépositions ont des traits communs, mais présentent des différences sémantiques et combinatoires fines : avec un ensemble les $X$, parmi vise les espaces entre les $\mathrm{X}$, alors que entre vise les $\mathrm{X}$ eux-mêmes et leur ordonnancement.

\section{Conclusion}

Après le rappel des points essentiels de notre argumentation en faveur de la reconnaissance de l'emploi spatial des prépositions ensemblistes qui oscillent entre la désignation du 'lieu' et du 'milieu' / 'environnement', il nous a semblé intéressant de détailler et de gloser les localisations du type 'environnement' de parmi et d'apporter ainsi des preuves linguistiques d'existence de localisations indéterminées, qui ne perdent pas leur spatialité à cause de leur imprécision. En bref, parmi signifie la dissociation et l'exploration interne d'un ensemble à frontières négligeables, les espaces unifiés délimités par les éléments de l'ensemble constituant un espace de forme variable qui permet soit la localisation indéterminée d'une ou de plusieurs entités quelque part ou çà et là, soit un ou des trajets sinueux explorant le continuum spatial protéiforme délimité par cet ensemble. Parmi crée par son sens l'idée d'une occupation partielle indéterminée de l'espace de type ensembliste ou l'idée de trajet(s) exploratoire(s) dans un ensemble d'éléments aléatoirement répartis.

\section{BIBLIOGRAPHIE}

Aurnague, M., 2004. Les structures de l'espace linguistique : regards croisés sur quelques constructions spatiales du basque et du français. Paris / Leuven : Peeters.

Aurnague, M. \& Stosic, D., 2002. « La préposition par et l'expression du déplacement : vers une catégorisation sémantique et cognitive de la notion de «trajet » ", Cahiers de Lexicologie 81/2, 113-139.

Bloch, O. \& von Wartburg, W., 1932 / 2008. Dictionnaire étymologique de la langue française. Paris : PUF.

Borillo, A., 1998. L'espace et son expression en français. Paris : Ophrys. 
Buridant, C., 2000. Grammaire nouvelle de l'ancien français. Paris : SEDES.

Cadiot, P., 2002. «Schémas et motifs en sémantique prépositionnelle : vers une description renouvelée des prépositions dites «spatiales » ", Travaux de linguistique 44, 9-24.

Fagard, B., 2010. Espace et grammaticalisation - L'évolution sémantique des prépositions dans les langues romanes. Sarrebruck : EUE, http://halshs.archives-ouvertes.fr/halshs-00637449/fr/.

Féraud, J.-F., 1787-1788. Le Dictionaire critique de la langue française informatisé de Jean-François Féraud, P. Caron \& L. Dagenas (eds), Université de Limoges, Université de Montréal, CNRS, ATILF, The ARTFL Project, http://dictionnaires.atilf.fr/dictionnaires/ FERAUD/index.html.

Gerhard-Krait, F. \& Kleiber, G., 2006. «Quelque part : du spatial au non spatial en passant par l'indétermination et la partition », Journal of French Language Studies 16 : 2, 147-166.

Gréa, P., 2005. «Les locutions prépositionnelles au milieu / centre de dans leur acception spatiale », Revue de Sémantique et Pragmatique 18, 87-112.

Guentcheva, Z., 2003. « Entre : préposition et préfixe », in P. Blumenthal \& J.-E. Tyvaert (eds), La cognition dans le temps. Tübingen : Max Niemeyer Verlag, 59-74.

Hilgert, E., 2007. « Étude de parmi. Le cas des tours partitifs », Scolia 21, 37-66.

Hilgert, E., 2010a. La partition et ses constructions en français, Genève : Librairie Droz.

Hilgert, E., 2010b. « Retour sur les prépositions ensemblistes à interprétation spatiale ", Scolia 24, 53-70.

Hilgert, E., 2010c. « La structure $X$ entre les $X$ : réduplication et exemplaire qualitativement saillant », in S.N. Osu, G. Col, N. Garric \& F. Toupin (eds), Construction d'identité et processus d'identification. Berne : Peter Lang, 135-148.

Homma, Y., 2009. «À propos de courir par la ville : emploi marginal et identité de la préposition par », in C. Guimier, J. François, E. Gilbert \& E. Krause (eds), Autour de la préposition. Caen : Presses Universitaires de Caen, 215-223.

Huyghe, R., 2009. Les noms généraux d'espace en français. Enquête linguistique sur la notion de lieu. Bruxelles : De Boeck \& Duculot.

Kwon-Pak, S.-N., 2006. « Entre vs. parmi : deux prépositions au centre de la partition », in G. Kleiber, C. Schnedecker \& A. Theissen (eds), La Relation partie-tout. Leuven : Peeters, 651-668.

Langacker, R., 1987. Foundations of Cognitive Grammar. vol. I. Theoretical prerequisites. Standford: Standford University Press.

Lemaréchal, A., 1998. Etude de morphologie en $f(x)$. Louvain / Paris : Editions Peeters.

Stosic, D., 2005. « Prendre par le sentier à travers le bois, ou comment à travers (se) fraie un chemin », in M. Tenchea \& A. Tihu (eds), Prépositions et conjonctions de subordination. Syntaxe et sémantique. Timisoara : Editura Excelsior Art, 207-218.

Vandeloise, C. 1988. «Les usages spatiaux statiques de la préposition à », Cahiers de Lexicologie 53 (2), 119-148.

Vandeloise, C., 2001. Aristote et le lexique de l'espace. Stanford : CSLI.

Vandeloise, C., 2004. «Quatre relations fondamentales pour la description de l'espace », Histoire Épistémologie Langage 26 / I, SHESL, 89-109. 


\section{NOTES}

1. Nous adoptons le point de vue de Borillo (1998: 89), qui inclut d'entre dans la liste des prépositions composées, au même titre que de sous, de derrière, jusqu'à, jusque sur, quelle que soit l'origine de la préposition de (exigée par un verbe, par une structure particulière, par le sens 'origine').

2. Définition de Lemaréchal (1998 : 202-204).

3. Cf. aussi Guentchéva (2003) qui identifie une interprétation ensembliste de entre, parallèlement à son emploi ternaire.

4. Cf. pour les emplois de celle-ci Le Grand Gaffiot (2000). Nous avons sélectionné deux exemples, l'un pour une interprétation spatiale, l'autre pour son sens ensembliste-partitif: (i) «inter falcarios CIC. Cat. 1, 8: au milieu des fabricants de faux = dans la rue des fabricants de faux "; (ii) « honestissimus inter suos CIC. Amer. 16 : le plus honorable parmi ses concitoyens ».

5. Fagard (2010 : 339) fait, à juste titre, le rapprochement entre parmi et enmi, préposition éphémère du français, grâce à leur formation par l'association de par ou en avec mi, du latin medius « qui est au milieu» (cf. aussi Bloch \& Wartburg, 1932 / éd. 2008, Buridant, 2000, parmi d'autres). L'étymologie permet de faire un lien entre parmi (par $+m i)$ et milieu $(m i+l i e u)$ de la locution au milieu de, qui sert de glose à parmi.

6. On trouve une information intéressante sur l'évolution des emplois de la préposition entre ensembliste dans le Dictionaire de Féraud (1787-1788: B112b), qui signale des emplois ne correspondant plus à son époque, assurés en revanche par parmi.

7. Voici quelques exemples d'opérations linguistiques sur des ensembles (cf. Hilgert (2010a) : a) parmi intervient dans l'expression de : (i) l'appartenance à un ensemble homogène : Ce restaurant se compte parmi les meilleurs restaurants de France; Paul est un étudiant parmi d'autres; (ii) l'affectation à un ensemble : On a classé à tort ce livre parmi les manuels; (iii) la sélection dans un ensemble d'individus à identité indéterminée : Il faudra choisir parmi nos salades du jour; (iv) la partition d'un ensemble, avec l'idée d'existence dans les limites de l'ensemble de départ d'un sous-ensemble différent dans le sens montré par le prédicat, ce sous-ensemble étant désigné généralement par un quantificateur: Parmi les propositions des entrepreneurs, [il y en a] deux / certaines / quelques-unes / plusieurs [qui] méritent d'être retenues [les autres ne méritent pas d'être retenues]; (v) l'application majoritaire de la prédication à un ensemble : La scarlatine a fait des ravages parmi les enfants ; b) entre ensembliste (les $N=$ plus de deux $\mathrm{N}$ ) intervient dans l'expression de : (i) l'appartenance à un ensemble hétérogène : J'ai lu Proust, entre autres [entre autres choses : j'ai lu aussi le Monde, je me suis également promené]; (ii) la sélection dans un ensemble d'individus à identité déterminée : Il faudra choisir entre le Petit Robert, le Larousse, le Littré et le Lexis ; (iii) la répartition entre les individus d'un ensemble : Il a partagé les bonbons entre les enfants ; c) d'entre ensembliste (les $N$ = plus de deux $\mathrm{N}$ ) aide à exprimer la partition d'un ensemble, avec l'idée d'extraction d'un sous-ensemble suite à un contraste prédicatif avec l'ensemble de départ: Dix d'entre les camarades se lèvent et vont vers D. et Thérèse (M. Duras, La douleur, 1985, p. 148) ; Deux ou trois d'entre les transfuges avaient été pris sur le fait (J. d'Ormesson, 1993, La Douane des mers, p. 447) ; Quelques-uns d'entre ont été pris sur le fait.

8. Si simple qu'il paraisse, ce critère n'a pas été utilisé par Kwon-Pak (2006), qui analyse injustement Il y a un papier parmi les livres comme un exemple de partition d'un ensemble, alors qu'il s'agit d'une localisation. De même, Guentchéva (2003: 69) traite globalement l'interprétation ensembliste de entre et ne distingue pas les cas d'appartenance à un ensemble (ex. 34) ou de partition d'un ensemble (ex. 32 et 35) de ceux de localisation d'une entité par rapport à un ensemble d'une autre catégorie que celle de la cible (ex. 31 et 33). 
9. Correspondant à la relation spatiale réalisée par dans et que Vandeloise $(2001,2004)$ appelle 'enveloppement'. Il mentionne au passage un cas d'enveloppement' dans X est entre $Y, Z$ et $W$.

10. L'idée de limites intrinsèques des éléments de l'ensemble est décisive pour distinguer, comme le fait Gréa (2005), le sens distributif de au centre de et collectif de au milieu de, mais elle passe au second plan dans le cas de au milieu de comparé à parmi, tous deux collectifs, où l'idée de limite floue de l'ensemble prend le dessus.

11. La préposition de exprime l'origine et existe dans la construction de verbes comme sortir, revenir, s'échapper, etc. En français contemporain, sortir de se combine avec entre et ne se combine pas avec parmi : sortir de parmi les arbres apparaît comme archaïsant.

12. Guentchéva (2003: 70) relève aussi des exemples d'utilisation de entre ensembliste en français classique où, « du point de vue synchronique, c'est parmi qui serait tout naturellement employé ». Cf. aussi Fagard (2010: 256) pour l'évolution des prépositions du français.

13. Un cas analogue : l'accumulation des SP spatiaux à sites 'single' constitués chacun d'une seule entité discrète, où les plus grands englobent les plus petits, comme dans La photo se trouve dans un album sur l'étagère de droite, dans la bibliothèque. Dans une phrase comme Elle y est, je l'ai vue, l'anaphorique $y$ ne peut renvoyer qu'à la place unique qu'occupe la photo et donc à l'ensemble des emplacements.

\section{RÉSUMÉS}

Le but de cet article est de montrer, dans un premier temps, la place de parmi dans le microsystème des prépositions ensemblistes en emploi spatial : parmi signifie la dissociation des éléments de l'ensemble localisateur et l'occupation partielle et aléatoire des espaces que ces éléments délimitent entre eux ; entre exprime l'ordonnancement des éléments de l'ensemble et la saturation de tous les espaces créés par des oppositions duelles démultipliées; la préposition composée d'entre indique le déplacement de la cible de l'intérieur vers l'extérieur de l'ensemblesite. On défend aussi l'idée que le site localisateur désigné par le SP introduit par une préposition ensembliste n'est pas multiple, comme cela a pu être affirmé, mais unique: le site, c'est la collection les $N$, que l'on se représente spatialement selon les prédications spécifiques à parmi, entre ou d'entre. Enfin, cette étude montre que l'imprécision localisatrice de parmi est la même que celle des diverses configurations correspondant à là, quelque part, çà et là, par ci par là, à travers, au milieu de et, enfin, dans cet environnement. L'étude de la représentation de l'espace avec parmi les $N$ conduit à l'idée que parmi signifie soit l'idée d'occupation partielle indéterminée, soit celle de trajet ou de réseau de trajets exploratoires, frayés dans un ensemble localisateur. Parmi signifie donc de multiples manières la dissociation et l'exploration interne d'un ensemble à frontières négligeables.

The purpose of this article is to show, first of all, the place of parmi in a specific micro-system of prepositions (which we call 'ensemblistes', i.e. "linking, adjoining") when used spatially : parmi refers to the dissociation of the elements belonging to the localizing set and the partial, yet random, occupation of the space delimited by those elements; entre shows the ordering of the elements belonging to the set and the saturation of all the spaces created by a demultiplied dual opposition ; the combination d'entre indicates that there has been a shift from the interior to the exterior of the site. The paper also supports the idea that the localizing site designated by the SP introduced by an adjoining preposition is not multiple, as it has been stated before, but unique : 
the site is, actually, a collection of Ns, which are spatially represented according to the predicates specific to parmi, entre and d'entre. Last but not least, this study shows that the imprecise localizing offered by parmi corresponds to various configurations consistent with à là, quelque part, çà et là, par ci par là, à travers, au milieu de and, ultimately, with dans cet environnement. The study of space representation with the help of parmi les $\mathrm{N}$ brings forth the idea that parmi signifies either the idea of partial and indeterminate occupation, or the one of exploring route or routes traced in a localizing set. In conclusion, parmi signals in several ways the dissociation and internal exploration of a set whose borders are negligible.

INDEX

Mots-clés : préposition, prépositions spatiales, prépositions ensemblistes, parmi, entre, d'entre Keywords : spatial prepositions, adjoining prepositions

\section{AUTEUR}

EMILIA HILGERT

Université de Reims 